\title{
'You wouldn't pass the salt' - from accusation to request; or the impact of learning context and learner identity on the acquisition of situational variation in highly advanced learners of English
}

\author{
Anne Marie Devlin \\ School of Languages, Literature and Cultures, UCC
}

\section{Introduction}

The field of second language acquisition is a fascinating and global topic. Many of us have spent long hours pouring over textbooks, memorising vocabulary and perfecting our grammar only to find that when we arrive in the country no-one understands us or our use of language unexpectedly gives rise to hilarity or even causes offense. We've been told, 'Don't worry. Spend a few weeks in the country and you'll soon soak up the language'. Again, this is not always the case. While many learners return from their time abroad showing and feeling huge improvements, there are others who seem not to have benefited from the experience. In fact, it is well known that people can live in another language environment for years and never 'pick up' the language. So, if learning doesn't necessarily help and spending time in the country doesn't always produce the desired results, then what are the factors that can help transform us from tongue-tied novices into eloquent conversationalists?

\section{Background}

In order to explore the factors, we need to look at the background to second language acquisition research.

For a long time, second language acquisition was thought to be the result of changing habits or acquiring new ones. All we needed to do was 'listen and repeat' and eventually we would get there. However, many realised that this explanation in no way accounted for two specific phenomena:

1. learners are capable of producing novel sentences i.e. sentences that they have not heard before

2. all learners make the same mistakes. 
To explain those phenomena, language acquisition researchers adopted a cognitive framework to the field. This trailblazing framework allowed a quantitative science to emerge which took the field out of the realm of habits into that of science. It was argued that we are all hardwired to acquire language, that we are all endowed with a 'language acquisition device' which ensures that we acquire a second language following a predetermined and fixed route. All learners will follow the same path irrespective of their first language (L1) or learning context i.e., where learning takes place. This route or path is known as interlanguage. This was revolutionary. It meant that language acquisition was systematic and could actually be analysed and quantified universally. The theory was underpinned by a series of studies into the systematic acquisition of grammatical features and developmental sequences such as forming questions and negatives. These studies showed, very compellingly, that there is a natural order of acquisition of grammatical features and that there are fixed stages to acquiring questions and negative forms. To use a metaphor, interlanguage is like a train. It has a fixed route and a fixed number of stations. When the train stops, it affords the passengers (learners) the opportunity to offload unnecessary baggage (old grammatical forms) and replace them with the newer forms.

As compelling as the argument for a purely cognitive framework is, it has several drawbacks. It presupposes that language is a monolith, i.e., that a language is a single, unchanging entity. It also presupposes a final destination (the native speaker) and if we reach that point further learning is neither necessary nor even possible. Likewise, it works on the premise the learner is a fixed, stable entity with no control over the journey; and that all learning contexts are equal. The train ploughs on regardless of external conditions. Moreover, one of the most depressing presuppositions is that learners seldom reach the final station of this journey with most leaving the train, or fossilising, some distance before the last stop.

However, since the late 1990's there has been a growing and convincing body of work advocating that language acquisition is, at least partially, socially constructed. Many now believe that language acquisition does not take place in a vacuum and that it is not necessarily a fixed process; rather, that it is dynamic and fluid and that the key elements in the process i.e., language, learner and context are also dynamic and fluid entities which change and develop over time and space forming a highly complex socio-cognitive ecosystem.

\section{Current Research Project}

The current research project aims to explore the dynamic relationship between these key entities and attempts to prove empirically that learning context and learner identity are crucial to the acquisition of the ability to vary language to suit the social or contextual situation by investigating the language of 20 non-native speaker teachers of English. 
The key questions posed are thus:

Is there a relationship between language proficiency level and a learner's ability to vary language to suit the situation?

Does time spent in the target language country aid the acquisition of variation in speech? If so, how much time is needed and how frequent should the visits be?

Does social and/or self-imposed identity act as a barrier or a conduit to the acquisition of variation?

In order to explain this point of view, we need to break down some of the beliefs held by those working from a purely cognitive point of view.

\section{Language as a Monolith}

The data used to underpin the interlanguage theory is based solely on grammatical aspects of language. However, it has long been acknowledged that language is more that a set of grammatical rules which we can categorise as correct or incorrect. It also has a very significant contextual/social aspect which can be varied to suit the situation.

There is no doubt that social context has a significant impact on what we say. It provides the speaker with a range of choices. Although such choices do not always impede comprehension, they can have serious effects on our relationships with people if the wrong selection is made. Just think of the impact on the listener of the following two questions:

What is your name?

Sorry, what was your name again?

The first question could be an imposition on the listener. It is reminiscent of an interview or even interrogation; while the second is more likely to put the listener at ease.

This area is known as sociolinguistic competence and while it has long been studied in first language, it is a relatively new area in second language acquisition studies. All aspects of language provide opportunities for variation. From the point of view of vocabulary, for example, when visiting the doctor we may refer to 'stomach' problems, but when talking to a child, the same problem becomes a sore 'tummy'.

In pragmatics - or how we do things with language, e.g. requesting, complaining, greeting, suggesting - this choice can be even more stark. Making the wrong choice can cause offense and can lead to negative stereotyping. It is not uncommon the hear, 'I don't like the (add a nationality of your choice). They are so rude!' Many of the (nationality of your choice) are not aware of how rude they sound in English, as a direct translation of the utterance would not have the same negative impact in their own language. Take for example, 'Girl, give me two coffees'. This kind of request would cause grave offense to a 
native English speaker, but would be a perfectly normal way of requesting for Russians both male and female. If we analyse our own use of English, the misunderstandings that can occur become apparent. Take for example the following exchange:
A: Are you OK?
B: I'm fine thanks

A perfectly innocent interaction? Move the context to an Irish pub and we have a disgruntled customer with the impression of the Irish as caring, but unwilling to take an order as s/he will not get the pint of Guinness and two gin and tonics required.

To sum up, it is clear that language is composed of so much more than a sequence of grammatical structures. The social/contextual aspect of language, which was once seen as peripheral, plays a pivotal role. It can therefore be surmised, that the notion of one fixed form of language does not hold true. What we have instead is a complex, dynamic blend of distinct varieties or discourse domains which come together to form a unified body. So, if this is true, how does a learner acquire the ability to vary language according to social/contextual demands? This question will be answered by exploring the following two elements.

\section{Learning Context}

Generally speaking learning context has been divided into 3 categories:

foreign language classroom

immersion classroom

study abroad

Traditional SLA research would posit that context has no impact on what is acquired. However, recent research into sociolinguistic competence has shown considerable differences in the nature of language acquired between classroom learners and study abroad learners in that study abroad learners acquire more variable aspects than classroom learners.

However, it is impossible to talk about all study abroad experiences as equal. In fact, research into the impact of language acquisition has produced very disparate results with some studies showing major improvements and others showing no real improvements when compared to 'at home' students. So, if all contexts are equal, how can this be the case? Well, there are many variables underlying study abroad. These include: duration of stay, intensity of contact with native speakers and accommodation. 


\section{Length Matters}

Recent research has been quick to point out the importance of intensity of contact over length of study abroad experiences. However, the current project is establishing a positive link between duration and intensity of contact. As part of my own research, I have looked at how contact with native speakers and type of language exchanges encountered is affected by length of time spent in the target language country. Results show that those who spend more than 1 year in the target language country are 5.5 times more likely to have exchanges with native speakers 'very often' than those who have spent 3 months or less. This gives rise to interesting findings regarding type of exchanges with native speakers. For those who spend a year or more, 50\% cite 'very often' transactional and interactional exchanges; whereas for those who spend 3 months or less, the figures are $16.7 \%$ and $8.3 \%$ respectively. The fact that half the learners encounter transactional and interactional language very often shows firstly, that length does matter and that, secondly, they are able to take part in a much wider range of discourse domains; that opportunities to 'be' and 'do' are much greater. This leads us on to the role of identity.

\section{Identity}

As already mentioned, those working from a purely cognitive point of view, see learner and native speaker identity as static entities in that they don't evolve or change. A learner is just a learner. However, this is very far from reality. Just like native speakers, non-native speakers are also a compound identity. By that I mean we are able to 'be' and 'do' a variety of identities. For example, we may 'be' a teacher 'doing' explaining a new concept to pupils. After that, we can change to 'being' a customer 'doing' asking for advice and later to 'being' a friend 'doing' gossiping. So, we can see that learner identity can and should be dynamic and to progress a learner should be afforded opportunities to develop these identities.

However, identity also plays another role. But this time it is self-imposed identity. That is the identity that the learner imposes on him/herself with regards to the second language. And just as we've seen that opportunities or lack of can have a serious effect on learners ability to develop a compound identity then a self imposed identity can act as a barrier or a conduit to language acquisition. If a learner positions him/herself as an outsider or fails to find a connection with the language or culture, this may prove a barrier to learning. Recent studies have shown how urban learners of the Irish language often fail to find a linchpin and often feel disenfranchised from this perceived rural language. In other words their lack of identification with the language can prove an almost insurmountable barrier. On the other hand being able to position yourself within the target culture may help with acquisition. 
So, to go back to the title of the article - 'You wouldn't pass the salt' - from accusation to request, we can see that the acquisition of the more social or variable aspects of the language require the complex and dynamic interaction between cognition, context and identity. The learner needs to have acquired the requisite grammatical knowledge to understand the sentence; however, the acquisition of grammatical forms is not enough to differentiate between the possible different meanings. To be able to accomplish this, the learner requires exposure to opportunities for exchanges across a wide range of discourse domains and variation in speech. This is best achieved through prolonged and intense contact with the native language. This, in turn, will lead to the development of compound identities where the learner can 'be' and 'do' as necessary; and finally, self-imposed identity as in where the learner positions him/herself in regards to the target language and culture.

Thanks to my supervisor, Dr Martin Howard. Anne Marie Devlin is student in the French Department under the supervision of Dr Martin Howard 